OPEN ACCESS

Edited by:

Antonio Ippolito,

University of Bari Aldo Moro, Italy

Reviewed by:

Alejandro Hernández,

University of Extremadura, Spain

Soner Soylu,

Mustafa Kemal University, Turkey

*Correspondence:

Yonghong Huang

gstshh@126.com

${ }^{\dagger}$ These authors have contributed equally to this work

Specialty section:

This article was submitted to

Food Microbiology,

a section of the journal

Frontiers in Microbiology

Received: 10 November 2021

Accepted: 07 January 2022

Published: 07 February 2022

Citation:

Sun M, Duan Y, Liu JP, Fu J and Huang Y (2022) Efficacy of Dimethyl Trisulfide on the Suppression of Ring

Rot Disease Caused by

Botryosphaeria dothidea and Induction of Defense-Related

Genes on Apple Fruits.

Front. Microbiol. 13:796167. doi: 10.3389/fmicb.2022.796167

\section{Efficacy of Dimethyl Trisulfide on the Suppression of Ring Rot Disease Caused by Botryosphaeria dothidea and Induction of Defense-Related Genes on Apple Fruits}

\author{
Meng Sun ${ }^{1,2,3,4 t}$, Yanxin Duan ${ }^{1,2,3,4 t}$, Jun Ping Liu'1,2,3,4, Jing Fu' ${ }^{1,2,3,4}$ and \\ Yonghong Huang 1,2,3,4*
}

${ }^{1}$ College of Horticulture, Qingdao Agricultural University, Qingdao, China, ${ }^{2}$ Laboratory of Quality and Safety Risk Assessment for Fruit (Qingdao), Ministry of Agriculture and Rural Affairs, Qingdao, China, ${ }^{3}$ National Technology Centre for Whole Process Quality Control of FSEN Horticultural Products (Qingdao), Qingdao, China, ${ }^{4}$ Qingdao Key Laboratory of Modern Agriculture Quality and Safety Engineering, Qingdao, China

Apple ring rot caused by Botryosphaeria dothidea is prevalent in main apple-producing areas in China, bringing substantial economic losses to the growers. In the present study, we demonstrated the inhibitory effect of dimethyl trisulfide (DT), one of the main activity components identified in Chinese leek (Allium tuberosum) volatile, on the apple ring rot on postharvest fruits. In in vitro experiment, $250 \mu \mathrm{L} / \mathrm{L}$ DT completely suppressed the mycelia growth of $B$. dothidea. In in vivo experiment, $15.63 \mu \mathrm{L} / \mathrm{L}$ DT showed $97 \%$ inhibition against the apple ring rot on postharvest fruit. In addition, the soluble sugar content, vitamin C content, and the soluble sugar/titratable acidity ratio of the DT-treated fruit were significantly higher than those of the control fruit. On this basis, we further explored the preliminary underlying mechanism. Microscopic observation revealed that DT seriously disrupted the normal morphology of $B$. dothidea. qRT-PCR determination showed the defense-related genes in DT-treated fruit were higher than those in the control fruit by 4.13-296.50 times, which showed that DT inhibited apple ring rot on postharvest fruit by suppressing the growth of $B$. dothidea, and inducing the defenserelated genes in apple fruit. The findings of this study provided an efficient, safe, and environment-friendly alternative to control the apple ring rot on apple fruit.

Keywords: Allium tuberosum, Botryosphaeria dothidea, biocontrol, defensive genes, fruit quality

\section{INTRODUCTION}

Apple is one of the most critical and popular fruits around the world. However, apple ring rot caused by the latent pathogen Botryosphaeria dothidea has seriously threatened apple production in recent years. The pathogen infects branches and trunks, resulting in wart-like symptoms around lenticels. As the disease aggravates, the infected trunks and shoots die. B. dothidea also infects apple fruit, causing slightly sunken lesions with alternating tan and brown rings. Subsequently, the diseased fruit rots quickly with a sour smell and oozes brown mucus sometimes (Tang et al., 2012; Bai et al., 2015). The decayed fruit proportion caused by the disease usually ranges between 
10 and 20\% each year. However, it may reach $70 \%$ in seasons with conditions conducive to fungal development (Zhao et al., 2016).

In addition to apple fruits, $B$. dothidea also infects various fruit such as fig (Ficus carica) (Wang et al., 2020), olive (Olea europaea L.) (Korukmez et al., 2019), sweet cherry (Prunus avium L.) (Zhang et al., 2019), pomegranate (Punica granatum L.) (Gu et al., 2020), apricot (Prunus armeniaca L.) (Huang et al., 2019b), mulberry (Morus alba L.) (Huang et al., 2019a), pear (Pyrus bretschneideri Rehd.) (Sun et al., 2020), avocado (Persea americana) (Qiu et al., 2020), and kiwifruit (Actinidia chinensis) (Wang et al., 2021). Therefore, it is indispensable to develop efficient ways to prevent and control the spread of B. dothidea.

Cultivating resistant varieties is the most economical and effective approach for controlling apple ring rot caused by B. dothidea. Unfortunately, the major commercial cultivars like red fuji, golden delicious, gala, and red delicious are highly susceptible to B. dothidea (Guan et al., 2015). Therefore, fungicide application is still the primary method to control apple ring rot worldwide (Fan et al., 2016, 2019). Previous studies showed that synthetic fungicides including fludioxonil, fluazinam, pyrisoxazole (Song et al., 2018), difenoconazole, tebuconazole, prochloraz, trifloxystrobin (Dai et al., 2017), tebuconazole (Fan et al., 2016), and pyraclostrobin (Fan et al., 2019) exhibit great potential for inhibiting the apple ring rot. However, the overuse of synthetic chemical pesticides leads to the development of fungicide resistance and causes environmental pollution and health problems.

Therefore, it increasingly stimulates the research on a safer and more eco-friendly alternative means to control plant disease. Naturally derived bioactive compounds are one of the most promising ecological alternatives and have advantages over synthetic fungicides. Previous studies showed that pure monoterpenes in plants including cuminaldehyde, geraniol, and $\beta$-citronellol have promising antifungal effects against B. dothidea (Zhang et al., 2018). In addition, 32 essential oil monomers exhibit a varying inhibitory effect on B. dothidea (Li et al., 2021). The lemon (Citrus limon L.) essential oil contains limonene (61.68\%), neral (21.66\%), $\gamma$-pinene $(10.23 \%), \gamma$-terpinene $(6.42 \%)$ and exhibits potent antifungal activity against $B$. dothidea (Ammad et al., 2018). 4-hydroxycinnamic acid from Moso bamboo (Phyllostachys pubescens) leaf shows good antifungal activity to $B$. dothidea (Liao et al., 2021). Matrine significantly inhibits the mycelial growth of B. dothidea (Pan et al., 2019). Chelidonine in Chelidonium majus, shows intense fungal activity against $B$. dothidea (Pan et al., 2017). Furocoumarins, phenylethyl esters, alcarindiol, and sesquiterpenoid from Notopterygium incisum exhibit antifungal activities against $B$. dothidea (Xiao et al., 2018). Several active components, including schisanhenol B, schizandrin A, schizarin D, schizandrin B, gomisin L, schizandrol A, schizandrol B, isoschizandrin, schisanlignone A, kadsulignan $\mathrm{M}$ identified from Schisandra chinensis, inhibit hyphal growth of $B$. dothidea and significantly inhibited the apple ring rot on fruits (Yi et al., 2016).

Our preliminary study found that the Chinese leek (Allium tuberosum) extract exhibited potent inhibition on the mycelia growth of $B$. dothidea, thereby significantly suppressing the incidence of apple ring rot on detached shoots and postharvest fruit (Zhao et al., 2017). However, the exact mechanism remains elusive. Therefore, in the present study, we tried to demonstrate the antifungal activity of dimethyl trisulfide (DT), one of the essential components in Chinese leek volatile, against apple ring rot on postharvest fruit. On this basis, we also attempted to explore the underlying mechanism involved in the DT inhibitory effect on apple ring rot from two aspects: fungus $B$. dothidea and the apple fruits.

\section{MATERIALS AND METHODS}

\section{Experimental Material, Fungus, and Reagents}

The apple fruit (Malus domestica Borkh. cv. Red Fuji) used in the experiments was purchased in the local supermarkets. The fruit with uniform sizes, no disease spots, and no mechanical damages was selected for the experiments. DT was provided by Cheng Du Micxy Chemical Co., Ltd., Chengdu, China. The fungal $B$. dothidea was isolated from diseased fruit in a apple orchards in Yantai city and identified by Sangon Biotech (Shanghai, China) (Fu, 2021), and it was kept in potato dextrose agar (PDA) medium.

\section{Determination of the Inhibitory Effect of Dimethyl Trisulfide on the Mycelia Growth of Botryosphaeria dothidea}

Various concentrations of DT $(250,125,62.5$, and $31.25 \mu \mathrm{L} / \mathrm{mL})$ were prepared to verify the inhibitory effects of DT on the growth of $B$. dothidea. The experiment was performed referring to the previously published method with minor modifications (Kurt et al., 2011). Firstly, a mycelial disk $(0.5 \mathrm{~cm}$ in diameter $)$ was inoculated in the center of a Petri dish $(9 \mathrm{~cm}$ in diameter, $70 \mathrm{~mL}$ in volume) containing $20 \mathrm{~mL}$ of PDA medium. After that, the various concentrations of DT $(100 \mu \mathrm{L})$ were added onto the filter paper $(2 \mathrm{~cm} \times 2 \mathrm{~cm})$ laid on the center of the Petri dish inner lid. Thus the actual DT concentrations in the Petri dish space were $500,250,125$, and $62.5 \mu \mathrm{L} / \mathrm{L}$, respectively. In addition, $100 \mu \mathrm{L}$ sterilized water was used as control. The experiment was performed in six replicates. The inoculated Petri dishes were inverted and incubated at $28^{\circ} \mathrm{C}$ in the dark for 5 days. The fungal colony diameters were measured every day to evaluate the inhibitory effect of DT on mycelia growth. The area-under-curve (AUC) of fungal diameter was calculated using the following formula (Huang et al., 2012).

$$
\mathrm{AUC}=\sum_{i=1}^{n-1}\left[\frac{X_{i+1}+X_{i}}{2}\right] t_{i+1}-t_{i}
$$

$X$ is the inhibition. $n$ is the number of evaluations, and the $\left(t_{i-1}-t_{i}\right)$ is the time interval (days) between two consecutive evaluations. 


\section{The Inhibitory Effects of Dimethyl Trisulfide on the Incidence of Apple Ring Rot on Fruit}

To assess the inhibitory effects of DT on the incidence of apple ring rot on fruit, we designed two experiments. The two experiments were the same except for the inoculation method.

\section{Experiment 1}

The healthy apple fruit was sterilized with $75 \%$ alcohol and then washed with sterilized distilled water three times. A mycelial disk $(0.5 \mathrm{~cm}$ in diameter) was inoculated into a small hole $(0.5 \mathrm{~cm}$ in diameter and $0.5 \mathrm{~cm}$ in depth) which was made at the fruit's equator with a sterilized hole puncher. Two inoculated fruits $(0.5 \mathrm{~L}$ in volume) were placed into plastic boxes $(4.5 \mathrm{~L}$ in volume). Then, $2 \mathrm{~mL}$ of DT at various concentrations $(125,62.5$, and $31.25 \mu \mathrm{L} / \mathrm{mL}$ ) were added to the petri dish lid placed at one of the corners of the plastic box. The same volume of sterilized water was used as a control. Thus the final DT concentration in the plastic box space was 62.5, 31.25 , and $15.63 \mu \mathrm{L} / \mathrm{L}$, respectively. The plastic boxes were sealed and incubated at $28^{\circ} \mathrm{C}$ in the dark. The experiment was repeated four times. The disease symptom was recorded, and the disease spot diameter was measured every day to evaluate the inhibitory effect of DT against apple ring rot. The AUC of disease spot diameters was calculated as the formula (1).

\section{Experiment 2}

Five mycelial disks $(0.5 \mathrm{~cm}$ in diameter $)$ were inoculated in $50 \mathrm{~mL}$ potato dextrose (PD) broth medium and incubated in a shaker at $28^{\circ} \mathrm{C}, 200 \mathrm{r} / \mathrm{min}$ for $48 \mathrm{~h}$. The obtained mycelium pellets were broken into homogenate by ultrasound. The healthy apple fruit was sterilized with 75\% alcohol and washed with sterilized distilled water three times. Then the fruit was inoculated with $B$. dothidea by dipping into the fungal homogenate for $15 \mathrm{~min}$. Two inoculated apple fruit $(0.5 \mathrm{~L}$ in volume) were placed in the plastic boxes $(4.5 \mathrm{~L}$ in volume). Then $2 \mathrm{~mL}$ of DT at various concentrations (125, 62.5 , and $31.25 \mu \mathrm{L} / \mathrm{mL}$ ) were added into the petri dish lid placed at one of the corners of the plastic box. The same volume of sterilized water was used as a control. Thus the final DT concentration in the plastic boxes was 62.5, 31.25, and $15.63 \mu \mathrm{L} / \mathrm{L}$, respectively. The plastic boxes were sealed and incubated at $28^{\circ} \mathrm{C}$ in the dark. The disease symptom was observed every day to evaluate the inhibitory effect of DT on apple ring rot.

\section{Effects of Dimethyl Trisulfide on the Mycelial Morphology of the Botryosphaeria dothidea}

A mycelial disk $(0.5 \mathrm{~cm}$ in diameter $)$ was inoculated on the center of the cellophane paper laid on the PDA medium $(20 \mathrm{~mL})$ contained in Petri dish ( $9 \mathrm{~cm}$ in diameter, $70 \mathrm{~mL}$ in volume). The Petri dishes were inverted and incubated at $28^{\circ} \mathrm{C}$ in the dark until the mycelium grew to half the medium surface. Then $100 \mu \mathrm{L}$ DT $(250 \mu \mathrm{L} / \mathrm{L})$ was added onto the filter paper $(2 \mathrm{~cm} \times 2 \mathrm{~cm})$ laid on the Petri dish's inner lid center. Thus, the actual DT concentration in the Petri dish space was $500 \mu \mathrm{L} / \mathrm{L} .100 \mu \mathrm{L}$ sterilized distilled water was used as a control. All the Petri dishes continued to be incubated at $28^{\circ} \mathrm{C}$ in the dark for $24 \mathrm{~h}$. Then the cellophane paper with mycelia was cut into 1 -cm-wide pieces and spread onto the glass slide. The mycelial morphology was observed using an inverted microscope (EVOS Auto 2, Thermo Fisher Scientific, San Jose, CA, United States) at $40 \times$.

\section{Effects of Dimethyl Trisulfide on the Internal Qualities of the Apple Fruit}

The experiment consisted of four treatments, viz. (1) CK: The apple fruit was soaked in PD broth medium for $15 \mathrm{~min}$. (2) Bd: The apple fruit was soaked in $B$. dothidea culture for $15 \mathrm{~min}$. (3) DT: The apple fruit was soaked in $62.5 \mu \mathrm{L} / \mathrm{L}$ DT for $15 \mathrm{~min}$. (4) $\mathrm{DT}+\mathrm{Bd}$ : The apple fruit was soaked in $62.5 \mu \mathrm{L} / \mathrm{L}$ DT for $15 \mathrm{~min}$, then were soaked in $B$. dothidea culture for $15 \mathrm{~min}$ following air-drying at room temperature for $1 \mathrm{~h}$. All the fruits were put into plastic boxes and incubated at $28^{\circ} \mathrm{C}$ in the dark. Apple fruit was sampled at $0,3,6,9$, and 12 days to determine the dynamic changes of internal fruit quality indices, including total soluble solid (TSS), soluble sugar (SS), titratable acidity (TA), and vitamin $\mathrm{C}$ (VC), total soluble solid/titrable acidity (TSS/TA), and soluble sugar/titrable acidity (SS/TA). TSS content was determined using a PAL-1 type sugar concentration detector (ATAGO, Japan). SS content was determined using the anthrone colorimetric method. The TA content was determined by $\mathrm{NaOH}$ titration. VC content was determined by 2, 6-dichloroindophenol colorimetric (Yang L. et al., 2020). The experiment was performed in three replicates. The AUC of internal quality indices was calculated as the formula (1), where $X$ is the contents of fruit internal quality indices.

\section{Determination of the Expression of Defense Related-Genes Responded to Dimethyl Trisulfide}

As previously described, four treatments (CK, DT, Bd, and $\mathrm{Bd}+\mathrm{DT}$ ) were performed. All the fruits were put into plastic boxes and incubated at $28^{\circ} \mathrm{C}$ in the dark. The disease symptoms were observed every day. 12 days later, a slight disease symptom emerged on the Bd-treated apple fruit. All the treated and control apple fruit were peeled around the equator with a sterilized paring knife. All the samples were stored at $-80^{\circ} \mathrm{C}$ for later use.

The expressions of the defense-related genes including phenylalanine ammonia-lyase (PAL), glucanase_1 (GLU-1), glucanase_2 (GLU-2), glucanase_3 (GLU-3), peroxidase_1 (POD-1), peroxidase_2 (POD-2), polyphenol oxidase (PPO), catalase (CAT), and endochitinase (CHI) were determined by quantitative real-time PCR (qRT-PCR). We drew the gene sequence information of the nine genes mentioned above from the apple genome (ASM211411v1). The special primers were designed using primer 5 (Table 1) and synthesized by Sangon Biotech (Shanghai) Co., Ltd., Shanghai, China.

According to RNAprep Pure Plant Plus Kit [Tiangen Biotech (Beijing) Co., Ltd., Beijing, China], the RNA was extracted from the fruit samples. And the cDNA was synthesized using HiScript ${ }^{\circledR}$ III RT SuperMix for qPCR (+gDNA wiper) (Nanjing Vazyme 
TABLE 1 | The primer sequences of defense-related genes and internal reference genes.

\begin{tabular}{|c|c|c|c|}
\hline GeneName & Gene ID & Primers & Sequence $\left(5^{\prime}-3^{\prime}\right)$ \\
\hline \multirow[t]{2}{*}{ PPO } & NM_001319261.1 & PPO -F & TCATGGCTCTTCTTCCCGTT \\
\hline & & PPO -R & GATTGGCAGATCGGAGCTTG \\
\hline \multirow[t]{2}{*}{$\mathrm{CHI}$} & NM_001293894.1 & $\mathrm{CHI}-\mathrm{F}$ & GAGACTACTGGAGGATGGGC \\
\hline & & $\mathrm{CHI}-\mathrm{R}$ & ATCCTITCCGATTGCTTGGC \\
\hline \multirow[t]{2}{*}{ CAT } & XM_008375181.3 & CAT-F & CCTCGTGGTITGCAGTGAA \\
\hline & & CAT-R & GGAAGGTGAACATGTGCAGG \\
\hline \multirow[t]{2}{*}{ POD-1 } & XM_029099288.1 & POD1-F & TCCTGTGCTGACATITTGGC \\
\hline & & POD1-R & AATCTACATITTGCCCGGCC \\
\hline \multirow[t]{2}{*}{ POD-2 } & XM_029091729.1 & POD2-F & GTTGCACTCGTGATGTTGGT \\
\hline & & POD2-R & CAATCATGGAAGTGGAGGCG \\
\hline \multirow[t]{2}{*}{ PAL } & XM_008368428.3 & PAL -F & GCAGAGCAACACAACCAAGA \\
\hline & & PAL -R & CGTTAAAGCCCATGGTGAGG \\
\hline \multirow[t]{2}{*}{ GLU-1 } & NM_001293850.1 & GLU1-F & GAGCCAGTGATTCAACGGAC \\
\hline & & GLU1-R & TGGAGGTTACTTCCAGGCAG \\
\hline \multirow[t]{2}{*}{ GLU-2 } & XM_008343526.2 & GLU2-F & ATGTGGTGGCATGTGAGAGA \\
\hline & & GLU2-R & CTAGCAAACCCGACATCAGC \\
\hline \multirow[t]{2}{*}{ GLU-3 } & XM_029095631.1 & GLU3-F & СССТGАTTCCAАССТTGСТG \\
\hline & & GLU3-R & AAATCCCTTGTCCGGTCCAT \\
\hline \multirow[t]{2}{*}{ Actin } & XM_008393049.3 & Actin-F & CTTCAATGTGCCTGCCATGTAT \\
\hline & & Actin-R & AATTCCCGTTCAGCAGTAGT \\
\hline
\end{tabular}

PPO, polyphenol oxidase; CHI, endochitinase; CAT, catalase; POD-1, peroxidase_1; $P O D-2$, peroxidase_2; PAL, phenylalanine ammonia-lyase; GLU-1, glucanase_1; GLU-2, glucanase_2; GLU-3, glucanase_3.

Biotech Co., Ltd., Nanjing, China). qRT-PCR was performed using ABI7500 Thermal Cycler (Applied Biosystems, Foster City, CA, United States) to detect the expressions of the nine defenserelated genes aforementioned. The reaction system $(10 \mu \mathrm{L})$ contained $5 \mu \mathrm{L}$ of $2 \times$ ChamQ SYBR Color qPCR Master Mix (Nanjing Vazyme Biotech Co., Ltd., Nanjing, China), $0.2 \mu \mathrm{L}$ of each primer, $0.2 \mu \mathrm{L}$ of the $50 \times$ ROX Reference Dye I, $1 \mu \mathrm{L}$ of cDNA, $3.4 \mu \mathrm{L}$ of the $\mathrm{ddH}_{2} \mathrm{O}$. The reaction conditions were as follows: $95^{\circ} \mathrm{C}$ for $30 \mathrm{~s}$, followed by 40 cycles of $95^{\circ} \mathrm{C}$ for $10 \mathrm{~s}$ and $60^{\circ} \mathrm{C}$ for $30 \mathrm{~s}$, then $95^{\circ} \mathrm{C}$ for $15 \mathrm{~s}, 60^{\circ} \mathrm{C}$ for $1 \mathrm{~min}$ and finally $95^{\circ} \mathrm{C}$ for $15 \mathrm{~s}$. Actin was used as the internal reference gene, and the relative expression was calculated by $2^{-\Delta \Delta C T}$ method (Livak and Schmittgen, 2001). The experiment was performed in three replicates.

\section{Statistical Analysis}

Experimental data were analyzed using standard analysis of variance (ANOVA) followed by least significant difference tests $(p<0.05)$ using the software statistical analytical system (SAS 9.0). Standard errors were calculated for all mean values.

\section{RESULTS}

\section{Dimethyl Trisulfide Inhibited the Mycelial Growth of Botryosphaeria dothidea}

The results showed DT strongly inhibited the mycelia growth of the $B$. dothidea. The mycelia growth of the control was initiated on the first day and rapidly grew on successive days.
On the fifth day, the fungal mycelia completely covered the whole surface of the Petri dish. But the DT-treated mycelia remained unchanged or grew slower than the control (Figure 1). Statistics showed that the colony diameters reduced with the increase of the DT concentration (Figure 2A). The inhibitory effect increased with DT concentration but decreased with incubation time. During the whole experimental period, 500 and $250 \mu \mathrm{L} / \mathrm{L}$ DT exhibited 100\% inhibition against $B$. dothidea. 125 and $62.5 \mu \mathrm{L} / \mathrm{L}$ DT showed 93.5 and $69.0 \%$ inhibition on the first day, which sharply decreased to 18.3 and $8.75 \%$ on the fifth day, respectively (Figure 2B). The AUC of fungal colony diameters treated by various DT concentrations were reduced by $18.9-100 \%$ compared to that of control $(P<0.0001)$ (Figure 2C).

\section{Dimethyl Trisulfide Disrupted the Mycelial Morphology of Botryosphaeria dothidea}

Under a microscope, the control mycelia had complete morphology with uniform thickness, smooth surface, slender and full shape (Figure 3A). However, DT seriously deformed the normal mycelial morphology. The outline of the DT-treated mycelia became blurred, with more bifurcation at the top, similar to the chicken feet. And the mycelia also became curved and wrinkled. The front end expanded, forming a tumor-like structure. Some mycelia ruptured and dissolved, and the contents spilled (Figures 3B-D).

\section{Dimethyl Trisulfide Inhibited the Incidence of Apple Ring Rot Disease Symptoms on Fruit Experiment 1}

The first 2 days after inoculation, disease spots gradually appeared around the control fruit's inoculating points. Three days later, the disease spots began to grow and developed rapidly. On the fifth day, the fruit was utterly rotten with a thick exudation on the surface and a sour smell. However, DTtreated apple fruit showed no disease symptoms during the early days of the experiment. After 5 days of inoculation, only mild disease spots appeared on the apple fruit treated with the low concentration DT $(15.63 \mu \mathrm{L} / \mathrm{L})$ (Figure 4). Statistical analysis showed that the disease spot diameter of the control fruit reached up to $10.03 \mathrm{~cm}$ on the fifth day, while that of fruit treated with a low DT concentration $(15.63 \mu \mathrm{L} / \mathrm{L})$ was $0.25 \mathrm{~cm}$ (Figure 5A), indicating a $97.5 \%$ inhibition. High DT concentrations (62.5 and $31.25 \mu \mathrm{L} / \mathrm{L})$ exhibited $100 \%$ inhibition against the apple ring rot (Figure 5B). The AUC of disease spot diameter on fruit treated by the various DT concentrations was reduced by $98.5-100 \%$ compared to that of control $(P<0.0001)$ (Figure 5C).

\section{Experiments 2}

Only 1 day after inoculation, sparse mycelial colonies with different sizes appeared on the control fruit. During the first week, the colonies successively emerged and enlarged quickly, finally covering the whole fruit surface. The disease 


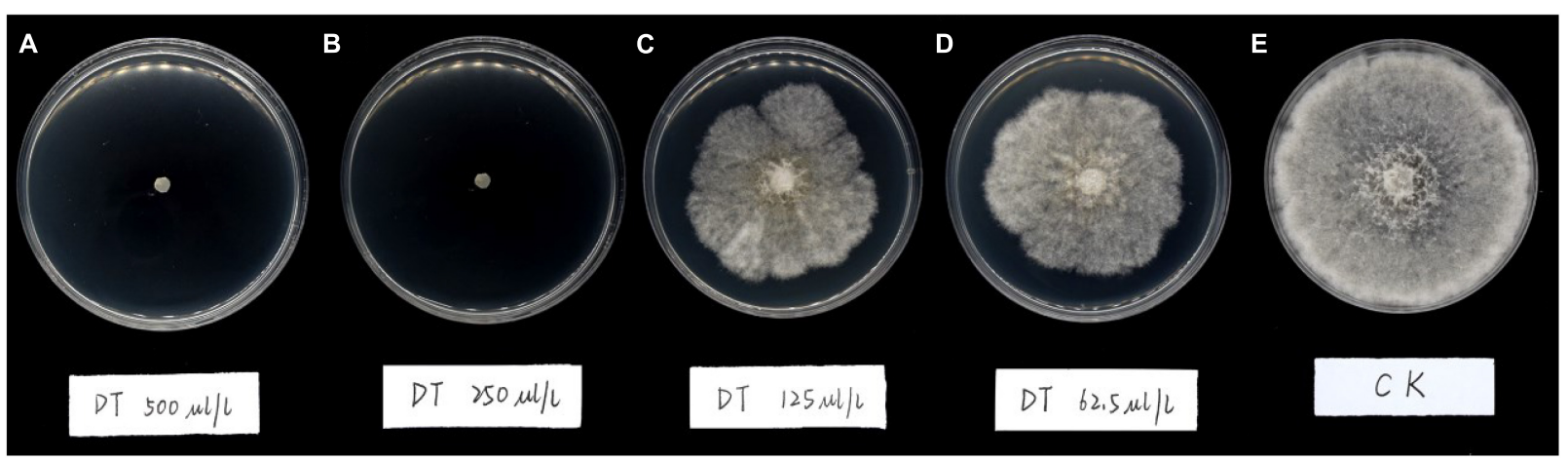

FIGURE 1 | The mycelial growth of Botryosphaeria dothidea on PDA medium containing various concentrations of dimethyl trisulfide for 5 days. (A) $500 \mu L / L$, (B) $250 \mu \mathrm{L} /$, (C): $125 \mu \mathrm{L} / \mathrm{L}$, (D) $62.5 \mu \mathrm{L} /$, (E) control.
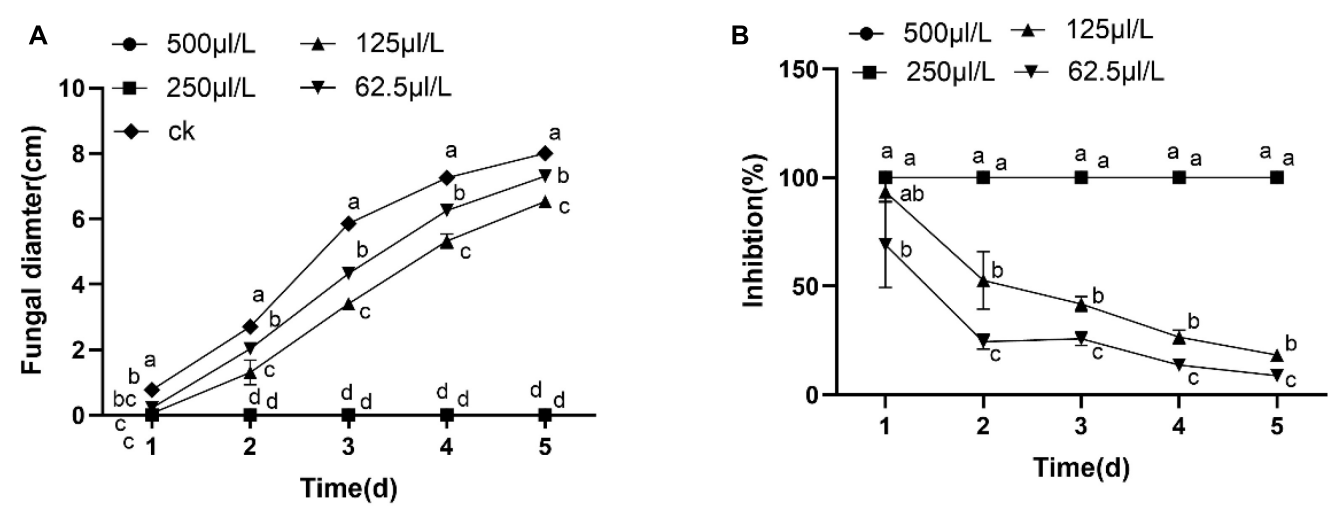

C

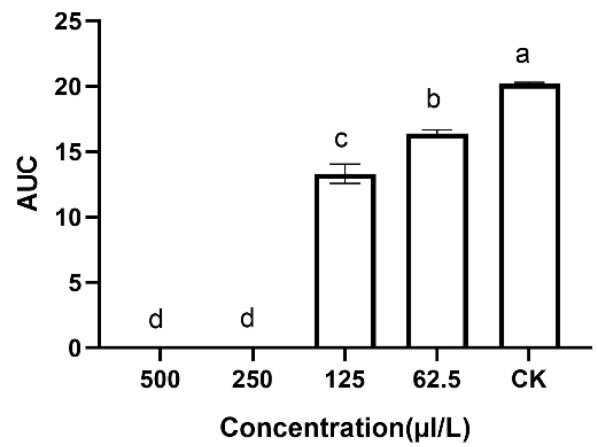

FIGURE 2 | The dimethyl trisulfide inhibition on the mycelia growth of Botryosphaeria dothidea. (A) The colony diameters of Botryosphaeria dothidea treated by the various concentration of dimethyl trisulfide. (B) The inhibition of various concentrations of dimethyl trisulfide against mycelia growth of Botryosphaeria dothidea. (C) The area-under-curve (AUC) of fungal colony diameters treated by various concentrations of dimethyl trisulfide. Different lowercase letters indicate a significant difference between treatments $(P<0.05)$.

spots gradually appeared across the fruit surface during the second week. They progressively expanded and joined together later, eventually leading to fruit decay during the third and fourth weeks. However, apple fruits treated with a low DT concentration $(15.63 \mu \mathrm{L} / \mathrm{L})$ showed no symptoms during the first week. During the second week, an average of 3 sparse mycelial colonies appeared on the fruit. On average, 10 sparse mycelial colonies appeared on the fruit during the third and fourth weeks. However, the apple fruit treated with higher
DT concentrations ( $>31.25 \mu \mathrm{L} / \mathrm{L}$ ) showed no obvious disease symptom and exhibited $100 \%$ inhibition against the apple ring rot throughout the experiment period (Figure 6).

\section{Dimethyl Trisulfide Enhanced the Apple Fruit Quality}

All the six fruit quality indices of the four treatments (CK, DT, $\mathrm{Bd}$, and $\mathrm{DT}+\mathrm{Bd}$ ) showed an up-and-down trend, fluctuating 


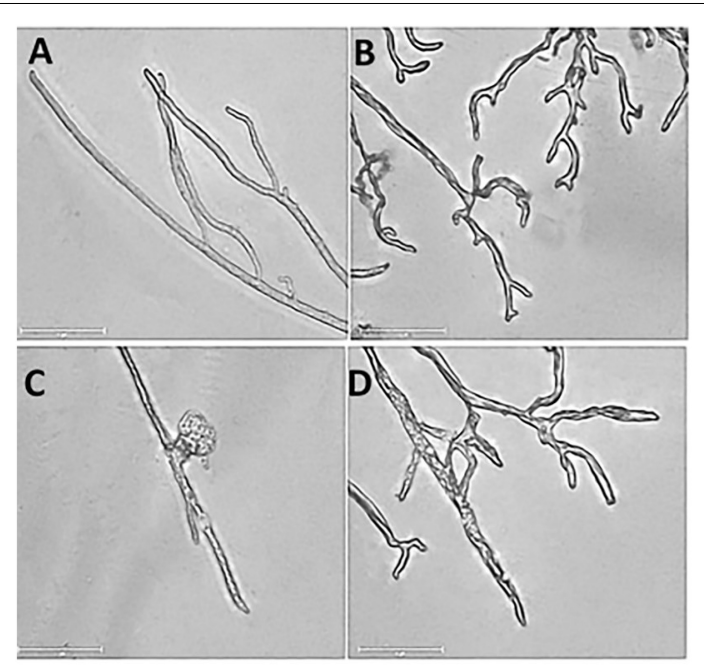

FIGURE 3 | The mycelial morphology of Botryosphaeria dothidea. (A) The mycelia of the untreated control. (B-D) The mycelia treated with $500 \mu \mathrm{L} / \mathrm{L}$ dimethyl trisulfide for $24 \mathrm{~h}$.

with prolonged time (Figure 7). The AUC of fruit quality indices was used to evaluate DT's overall effect on the fruit quality. Compared to the control fruit (CK), DT treatment (DT) increased the SS content, SS/TA ratio and VC content by $4.22 \%$ $(P=0.0124), 16.59 \%(P=0.0209)$, and $109.80 \%(P<0.0001)$, respectively. Compared to the fruit inoculated with $\mathrm{Bd}(\mathrm{Bd})$, DT treatment (DT + Bd) significantly increased VC content by $86.13 \%(P=0.0499)$, SS content by $3.67 \%(P=0.0346)$, and also enhanced SS/TA ratio by $19.05 \%(P=0.0581)$ (Figure 8$)$.

\section{Dimethyl Trisulfide Induced the Expression of Defense-Related Genes}

Dimethyl trisulfide induced all the detected genes in apple fruit, six (GLU-1, GLU-2, POD-1, POD-2, CHI, and CAT) of which were significantly up-regulated (Figure 9). Compared with the control (CK), the six genes in DT-treated apple fruit (DT) were up-regulated by 14.91 (GLU-2) to 84.09 (CAT) times, with an average of 39.09 times. Compared to fruit inoculated with $B$. dothidea $(\mathrm{Bd})$, the six genes in DT-treated apple fruit
$(\mathrm{Bd}+\mathrm{DT})$ were increased by $4.13(\mathrm{CHI})$ to 296.50 (POD-2) times, averaged 95.24 times. These results revealed that the DT markedly induces apple fruit's defense-related genes whether or not they were inoculated with $B$. dothidea.

\section{DISCUSSION}

Nowadays, extensive application of various synthetic fungicides is still the primary management strategy that effectively controls apple ring rot. But the pesticide residue resulted in potential harm to the environment, animals, even humans. Therefore, due to natural products' environment-friendly and low toxicity, developing natural fungicides from plants has attracted more attention worldwide. In the present study, we revealed that DT, one of the main components from Chinese leek, significantly suppressed apple ring rot on postharvest fruit. DT naturally existed in Chinese leek and other Allium plants. Plants of the Allium family, such as garlic (Allium sativum), onion (Allium cepa), and Chinese leek, have been cultivated for food since earliest times (Munday et al., 2003). And DT is widely distributed in foods and beverages such as broccoli, milk, cheese, whiskey, hineka, beer, and wine (Gijs et al., 2002; Isogai et al., 2009). Therefore, using DT to control apple ring rot is efficient and safe for humans, animals, and the environment, providing a possible way to control apple ring rot.

In the present study, we found that the DT inhibition at low concentration on the mycelia growth sharply decreased with the prolonged experimental time. For example, 125 and $62.5 \mu \mathrm{L} / \mathrm{L}$ DT showed 93.5 and $69.0 \%$ inhibition on the first day, but they decreased to 18.3 and $8.75 \%$ on the fifth day, respectively. That is because DT was highly volatile and quickly escaped from the Petri dishes into the air in a short period. In addition, DT interacted with the fungal mycelia and partly decomposed. The escape or decomposition rapidly decreased DT concentration in the petri dish. However, the remaining concentration was not sufficient to inhibit mycelial growth. To solve this problem in practical application, DT can be prepared into the slow-release formulation to maintain sustainable high inhibition.

To prove the inhibitory effect of DT on apple ring rot on postharvest fruit, we designed two inoculation methods, inoculating the apple fruit with a mycelial disk and socking

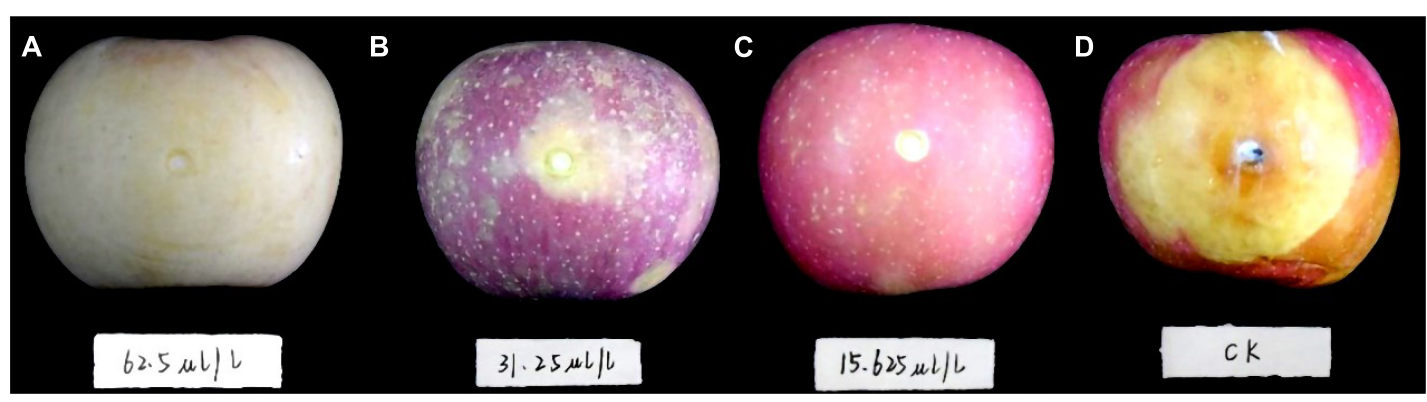

FIGURE 4 | The apple fruit was first inoculated with Botryosphaeria dothidea disk, subsequently treated with dimethyl trisulfide, then cultured at $28^{\circ} \mathrm{C}$ in the dark for 5 days. Apple fruits with different treatments exhibited varying degrees of disease symptoms. (A) $62.5 \mu \mathrm{L} / \mathrm{L}$, (B) $31.25 \mu \mathrm{L} / \mathrm{L},(\mathbf{C}) 15.63 \mu \mathrm{L} / \mathrm{L}$, (D) control. 

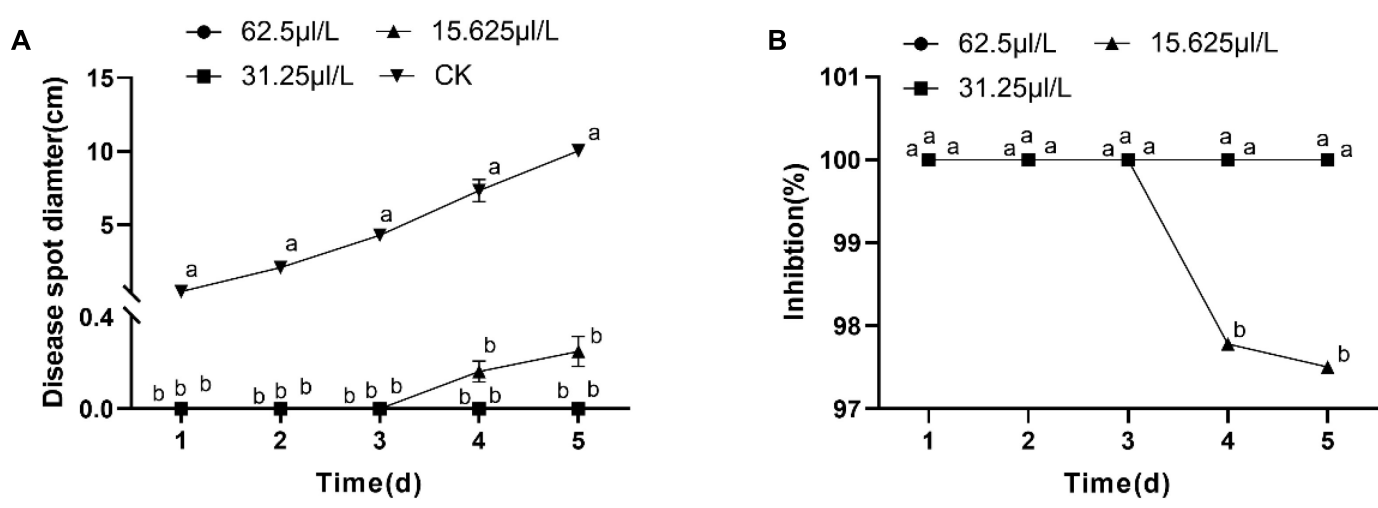

C

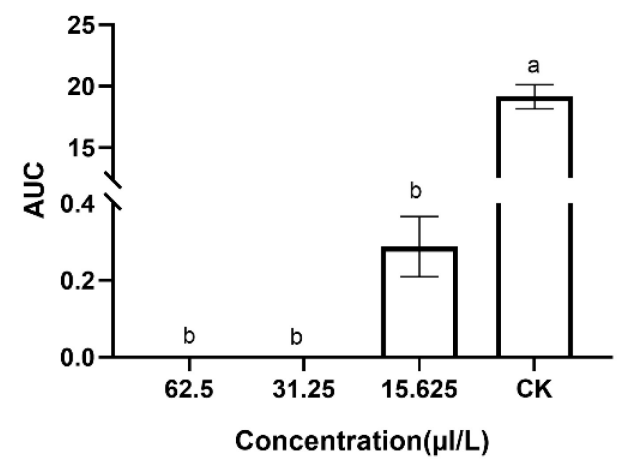

FIGURE 5 | The inhibitory effect of dimethyl trisulfide on the apple ring rot on apple fruit. (A) The disease spot diameter on apple fruits treated by various concentrations of dimethyl trisulfide. (B) The inhibition of various concentrations of dimethyl trisulfide against the apple ring rot on fruit. (C) The area-under-curve (AUC) of disease spot diameter on fruit treated by the various concentration of dimethyl trisulfide. Different lowercase letters indicate a significant difference between different treatments $(P<0.05)$.

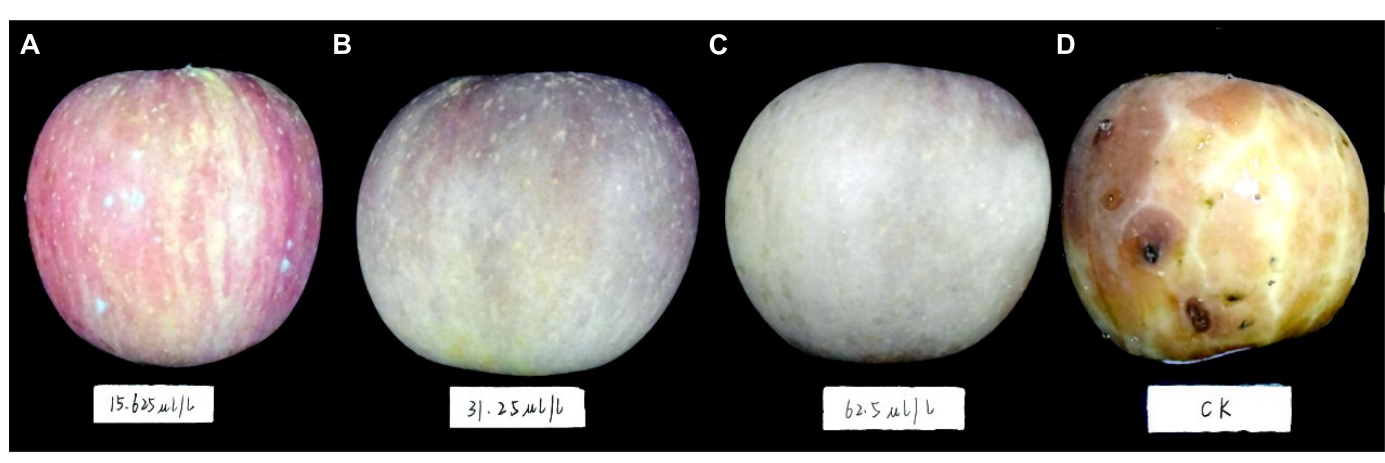

FIGURE 6 | The apple fruit was first dipped in a Botryosphaeria dothidea culture for $15 \mathrm{~min}$, subsequently exposed to various concentrations of dimethyl trisulfide, then cultured at $28^{\circ} \mathrm{C}$ in the dark for 4 weeks. Apple fruits with different treatments exhibited varying degrees of disease symptoms. (A) $15.63 \mu \mathrm{L} / \mathrm{L}$, (B) $31.25 \mu \mathrm{L} / \mathrm{L}$, (C) $62.5 \mu \mathrm{L} / \mathrm{L}$, (D) control.

the apple fruit in the $B$. dothidea culture for $15 \mathrm{~min}$. The two experiments revealed that DT significantly suppressed the incidence of the disease. The high concentration (62.5 and $31.25 \mu \mathrm{L} / \mathrm{L})$ of DT completely inhibited the disease, and the low concentration $(15.63 \mu \mathrm{l} / \mathrm{L})$ also showed more than $90 \%$ inhibition. However, the red skin color of apple fruit treated with high concentration (62.5 and $31.25 \mu \mathrm{L} / \mathrm{L})$ DT somewhat faded, but that of the apple fruits treated with low concentration
(15.63 $\mu \mathrm{l} / \mathrm{L})$ DT was perfectly maintained. Therefore, we suggest using lower DT concentration to treat apple fruit in practical application, dramatically maintaining the fruit color and preventing disease. Furthermore, the pathogen amount carried on the field fruit in the practical production was far lower than those artificially inoculated in our experiment. Thus, our present study demonstrated the potential application of DT as an alternative strategy against apple ring rot caused by $B$. dothidea. 

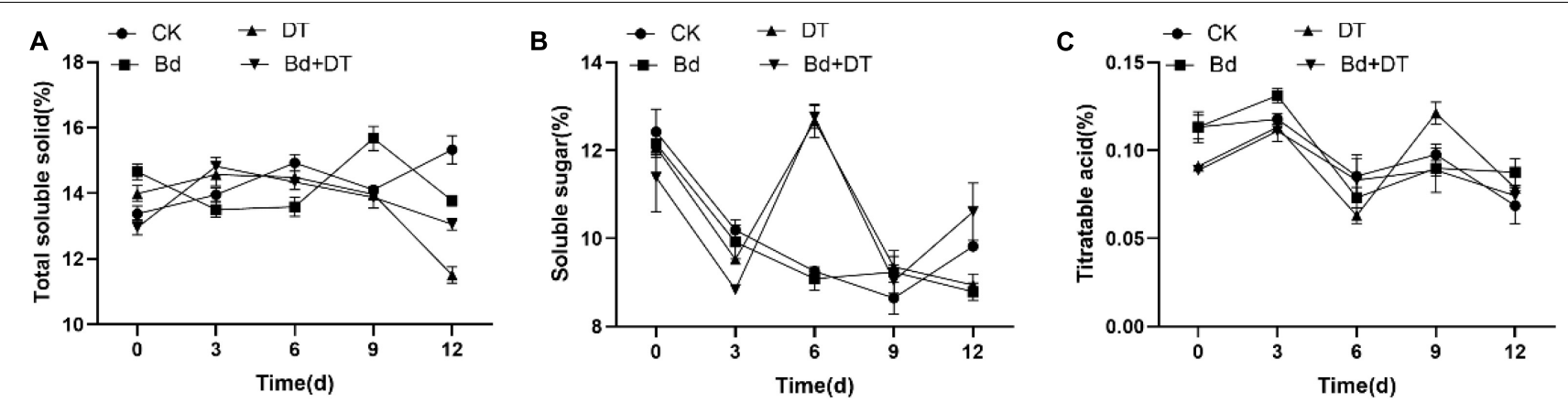

D

E
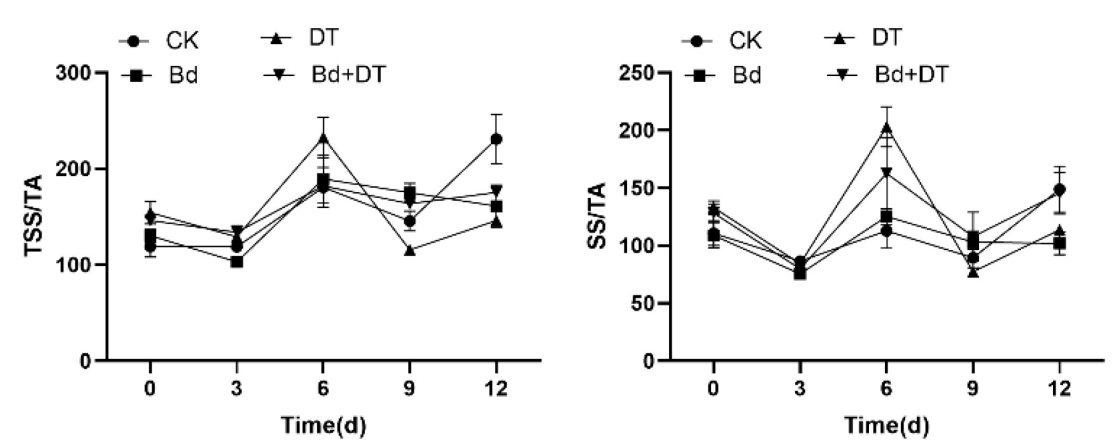

$\mathbf{F}$

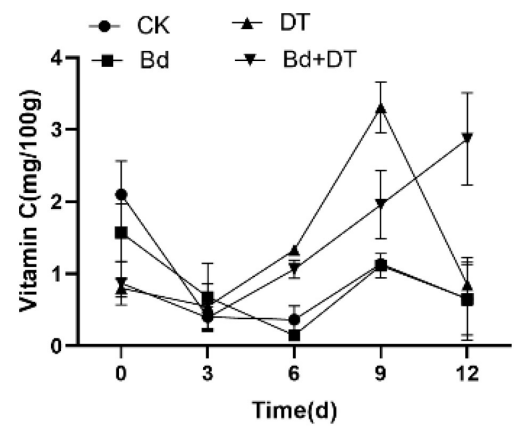

FIGURE 7 | The dynamic changes of internal quality indexes including total soluble solid (A), soluble sugar (B), titratable acidity (C), TSS/TA (D), SS/TA (E), and vitamin $C(\mathbf{F})$ in the different treatment fruit.

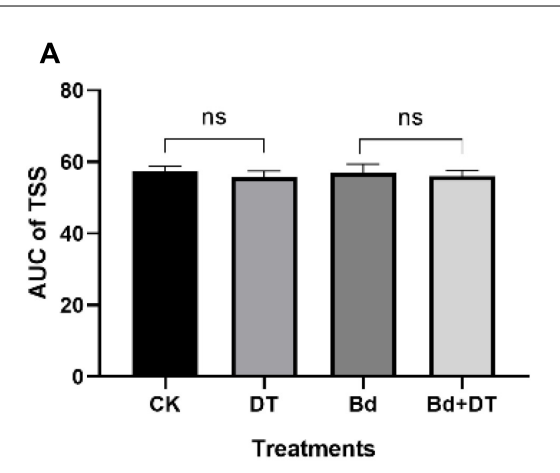

D

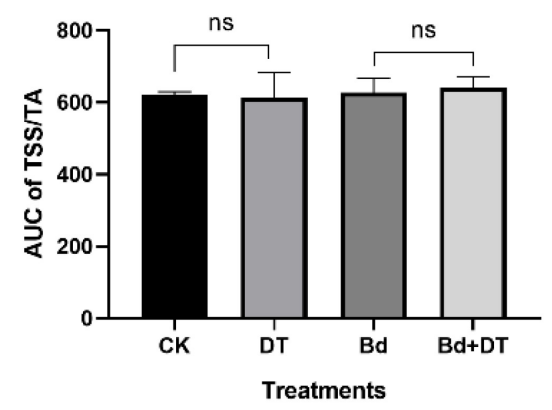

B

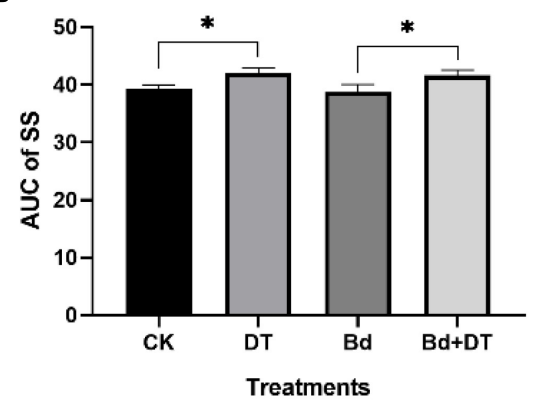

E

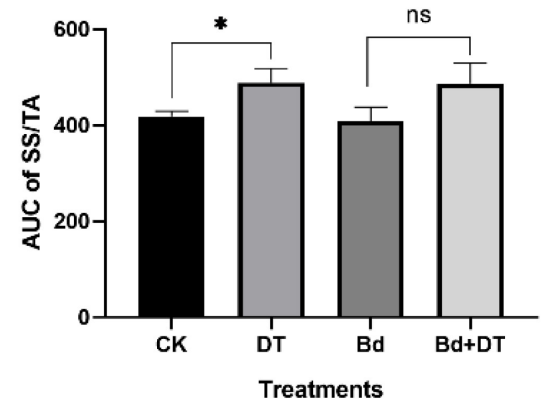

C

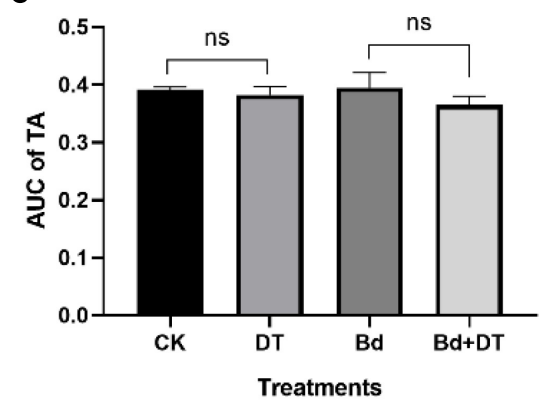

$\mathbf{F}$

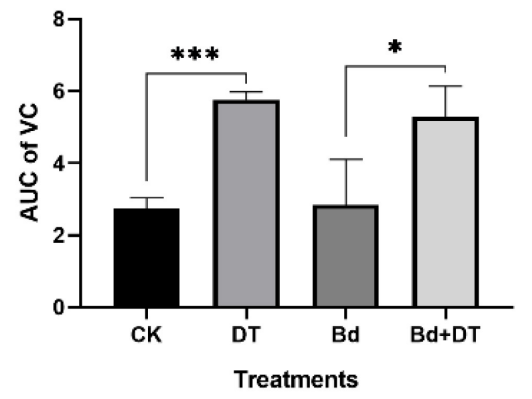

FIGURE 8| The area-under-curve (AUC) of internal quality indexes including total soluble solid (A), soluble sugar (B), titratable acidity (C), TSS/TA (D), SS/TA (E), and vitamin $\mathrm{C}(\mathbf{F})$ the different treatment fruit. ${ }^{*} P<0.05$, ${ }^{* * *} P<0.001$, ns $P>0.05$. 
A

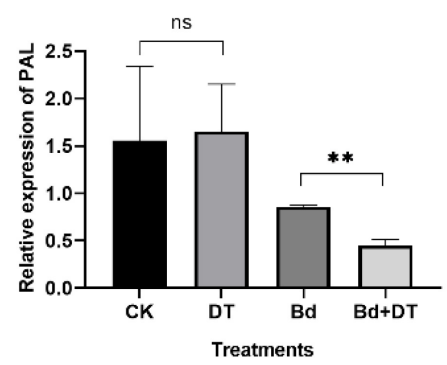

D

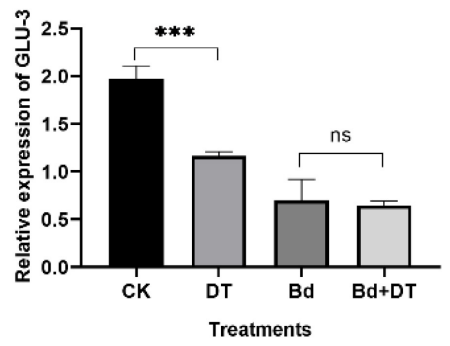

G

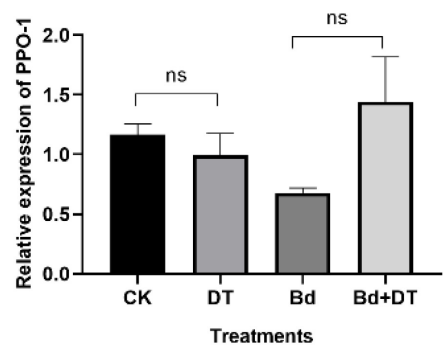

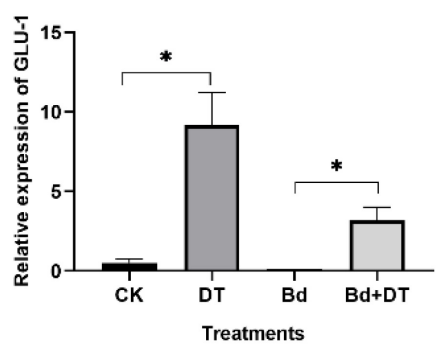

E

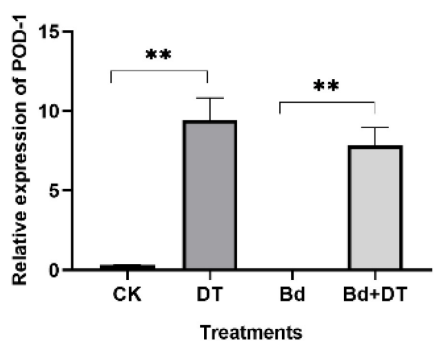

H

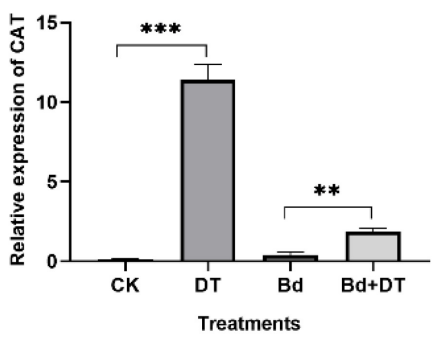

C

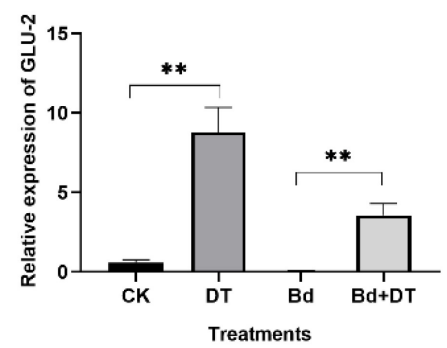

F

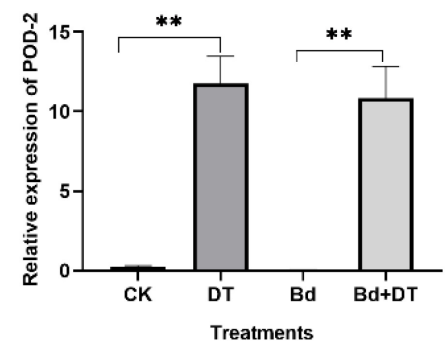

I

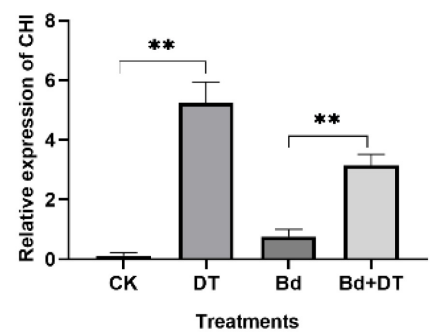

FIGURE 9 | The expression analysis of various defense-related genes including phenylalanine ammonia-lyase (A), glucanase_1 (B), glucanase_2 (C), glucanase_3 (D), peroxidase_1 (E), peroxidase_2 (F), polyphenol oxidase (G), catalase $\mathbf{( H )}$, endochitinase $(\mathbf{I})$ in the DT-treated fruit. ${ }^{*} P<0.05,{ }^{* *} P<0.01,{ }^{* * *} P<0.001$, ns $P>$ 0.05 .

The present study explored the underlying mechanism from the fungal B. dothidea aspect and the apple fruit aspect. From the findings in the present study, we deduced that DT inhibits the disease incidence by suppressing the growth of $B$. dothidea and inducing defense-related genes in apple fruit.

On the one hand, DT caused severe disruption to $B$. dothidea mycelia, leading to its slow growth or even death, further decreasing its vigor and amounts, thereby reducing the infection probability and ultimately suppressing the incidence of apple ring rot on apple fruit. The previous study showed that the essential oil aromatic plants, including origanum (Origanum syriacum L.), lavender (Lavandula stoechas L.), and rosemary (Rosmarinus officinalis L.), significantly inhibit the growth of Phytophthora infestans (Soylu et al., 2006) and Botrytis cinerea (Soylu et al., 2010) by causing considerable morphological degenerations of the fungal mycelia. Origanum contains carvacrol (79.8\%) and p-cymene $(8.2 \%)$, lavender contains camphor $(20.2 \%), 1,8$-cineole (35.5\%), $\alpha$-thujone $(15.9 \%)$ and fenchone $(13.5 \%)$, rosemary contains borneol (20.4\%), camphor
(19.5\%), 1,8-cineole (17.4\%) and linalool (6.1\%) as the main components (Soylu et al., 2006). These components interfere with the enzymatic reactions of wall synthesis, resulting in considerable morphological alterations in mycelia such as cytoplasmic coagulation, vacuolations, hyphal shriveling, and protoplast leakage, finally affecting the fungal morphogenesis and growth (Rasooli et al., 2006; Soylu et al., 2006, 2010). Therefore, we believe the direct effect of DT on fungal $B$. dothidea mycelium may be an important factor inhibiting the ring rot disease on apple fruit.

On the other hand, DT significantly induced defense-related genes, including GLU-1, GLU-2, POD-1, POD-2, CHI, and CAT, by 4.13 to 296.49 times compared to the control. Previous studies revealed that the defense-related response played an essential role in improving the disease resistance of plants. GLU enhances the tolerant of rough lemon rootstock against foot rot (Sandhu et al., 2019), improves the inhibition of rice against Rhizoctonia solani (Kumar et al., 2018), reduces the rice sheath blight (Kaur et al., 2021), and confers tea tree 
resistance against blister blight disease (Singh et al., 2018). POD is involved in the resistance response of coffee varieties to Colletotrichum kahawae (Diniz et al., 2019), potato to P. infestans (Yang Y. et al., 2020), Arabidopsis thaliana to Botrytis cinerea, Colletotrichum higginsianum, and Pectobacterium carotovorum (Zhao et al., 2019), Citrus sinensis to citrus bacterial canker (Li et al., 2020). CAT contributes maize to resistance against maize chlorotic mottle virus infection (Jiao et al., 2021), Nicotiana tabacum against Chilli veinal mottle virus infection (Yang $\mathrm{T}$. et al., 2020). CHI enhances tobacco tolerance against $B$. cinerea (Navarro-Gonzalez et al., 2019), Arabidopsis thaliana resistance against the bacterium Xanthomonas campestris pv. campestris (Xcc) (Santos et al., 2019), and tomato resistance against B. cinerea (Zheng et al., 2018). According to the above studies, the significantly up-regulated POD, GLU, CHI, and CAT were probably critical in enhancing apple fruit resistance against $B$. dothidea infection.

In practical application, we can spray DT on the tree or the fruit during the growing season to control apple ring rot on branches or fruit. After harvesting, we can spray DT on fruit, dip the fruits in DT solution, or place DT in the corner of the fruit box to prevent apple ring rot on postharvest fruit. Chinese leek contains a large amount of DT or other organic sulfides. The essential oil of Chinese leek leaves contains disulfide compounds (64.9\%) and trisulphide compounds (18.9\%), the flower contains trisulphide compounds (34.0\%) and disulfide compounds $(20.2 \%)$, the essential oil of rhizome contains trisulphide compounds (47.3\%), and the seed contains disulfide $(32.4 \%)$ and tetrasulfide $(5.2 \%)$ as the main constituents ( $\mathrm{Hu}$ et al., 2013). These sulfides contained in Chinese leek, including allyl methyl sulfide, methyl disulfide, dimethyl trisulfide, and methyl allyl trisulfide (Zhao et al., 2017), diallyl disulfide, diallyl trisulfide, and diallyl tetrasulfide (Rattanachaikunsopon and Phumkhachorn, 2009), also exhibit vigorous and widely antifungal or antibacterial activity. Our previous study showed that Chinese leek significantly reduced the banana Fusarium wilt (Huang et al., 2012) by intercropping or rotating crops. Therefore, we can also intercrop Chinese leek in the orchard to control apple ring rot in practice production.

Besides Chinese leek, other Allium plants, including A. sativum (Natasya-Ain et al., 2018), A. cepa (Balamanikandan et al., 2015; Ortega-Ramirez et al., 2017), Allium stipitatum (Karunanidhi et al., 2017), Allium saralicum (Zangeneh M.M. et al., 2019), Allium noeanum (Shahriari et al., 2019), Allium saralicum (Zangeneh A. et al., 2019), Allium hirtifolium (Ismail et al., 2013), are reported to possess extensive antifungal or antibacterial activity. They also contain a large number of organic sulfides with intense antifungal activity. For example, propyl-propane thiosulfinate, propyl-propane thiosulfonate (Sorlozano-Puerto et al., 2020), methyl allyl thiosulphinate, E-ajoene and Z-ajoene from garlic (A. sativum), elephant garlic (Allium ampeloprasum), and onion (A. cepa) (Hughes and Lawson, 1991), Allicin (Bhattacharya et al., 2019), diallyl disulfide (Jin et al., 2021) from garlic (A. sativum), also exhibit vigorous and widely antifungal or antibacterial activity.
These Allium plants are an excellent natural resource to control plant disease. However, they have been ignored for many years. For example, after harvesting garlic bulbs, the plant is often discarded in the field bank and the ditch, resulting in waste and pollution. We can take good advantage of these valuable resources by burying them in the soil around the tree, which controls the disease incidence and reduces pollution.

Perfect measures to control postharvest fruit diseases must have two fundamental characteristics: to effectively control disease occurrence and maintain fruit quality. In the present study, we demonstrate that DT showed a strong inhibitory effect on apple ring rot and significantly enhances SS and VC contents and the SS/TA ratio of the apple fruit, which suggests that DT is an ideal alternative strategy against postharvest apple ring rot.

\section{CONCLUSION}

In the present study, we demonstrated that DT, the main component of Chinese leek, significantly suppresses the mycelia growth of $B$. dothidea and inhibits the incidence of apple ring rot on postharvest fruit. Most importantly, DT significantly enhanced the soluble sugar content, vitamin C content, and soluble/titratable acidity ratio. We further found DT inhibited the apple ring rot by directly suppressing the pathogen growth and inducing the fruit's defense-related response. Therefore, it provides an efficient, safer, and environment-friendly alternative to control the apple ring rot on apple fruit.

\section{DATA AVAILABILITY STATEMENT}

The original contributions presented in the study are included in the article/supplementary material, further inquiries can be directed to the corresponding author.

\section{AUTHOR CONTRIBUTIONS}

$\mathrm{YH}$ contributed to the conception of the study and revised the manuscript. MS and YD wrote the manuscript, performed the experiments, and collected the data. JF experimented and collected the data. JL performed qRT-PCR validation. All authors contributed to the article and approved the submitted version.

\section{FUNDING}

This work was supported by the National Natural Science Foundation of China (31471864), the Natural Science Foundation of Shandong Province (ZR2020MC143), and the Qingdao Agricultural University High-level Personnel Startup Fund, China (6631115024). 


\section{REFERENCES}

Ammad, F., Moumen, O., Gasem, A., Othmane, S., Hisashi, K. N., Zebib, B., et al. (2018). The potency of lemon (Citrus limon L.) essential oil to control some fungal diseases of grapevine wood. Comptes Rendus Biol. 341, 97-101. doi: 10.1016/j.crvi.2018.01.003

Bai, S., Dong, C., Zhu, J., Zhang, Y., and Dai, H. (2015). Identification of a xyloglucan-specific endo-(1-4)-beta-D-glucanase inhibitor protein from apple (Malus $\mathrm{x}$ domestica Borkh.) as a potential defense gene against Botryosphaeria dothidea. Plant Sci. 231, 11-19. doi: 10.1016/j.plantsci.2014.11.003

Balamanikandan, T., Balaji, S., and Pandiarajan, J. (2015). Biological synthesis of silver nanoparticles by using onion (Allium cepa) extract and their antibacterial and antifungal activity. World Appl. Sci. J. 33, 939-943. doi: 10.5829/idosi.wasj. 2015.33.06.9525

Bhattacharya, S., Sen, D., and Bhattacharjee, C. (2019). In vitro antibacterial effect analysis of stabilized PEGylated allicin-containing extract from Allium sativum in conjugation with other antibiotics. Process Biochem. 87, 221-231. doi: 10. 1016/j.procbio.2019.09.025

Dai, D. J., Wang, H. D., Wang, Y. P., and Zhang, C. Q. (2017). Management of Chinese hickory (Carya cathayensis) trunk canker through effective fungicide application programs and baseline sensitivity of Botryosphaeria dothidea to trifloxystrobin. Austral. Plant Pathol. 46, 75-82. doi: 10.1007/s13313-0170465-4

Diniz, I., Azinheira, H., Figueiredo, A., Gichuru, E., Oliveira, H., GuerraGuimarães, L., et al. (2019). Fungal penetration associated with recognition, signaling and defence-related genes and peroxidase activity during the resistance response of coffee to Colletotrichum kahawae. Physiol. Mol. Plant Pathol. 105, 119-127. doi: 10.1016/j.pmpp.2017.12.005

Fan, K., Wang, J., Fu, L., Li, X., Zhang, Y., Zhang, X., et al. (2016). Sensitivity of Botryosphaeria dothidea from apple to tebuconazole in China. Crop Protect. 87, 1-5. doi: 10.1016/j.cropro.2016.04.018

Fan, K., Wang, J., Fu, L., Zhang, G. F., Wu, H. B., Feng, C., et al. (2019). Baseline sensitivity and control efficacy of pyraclostrobin against Botryosphaeria dothidea isolates in China. Plant Dis. 103, 1458-1463. doi: 10.1094/PDIS-0718-1214-RE

$\mathrm{Fu}$, J. (2021). Studies on the control and mechanism of volatiles from Chinese leek against apple ring rot. $\mathrm{Ph}$. D. thesis. Qingdao: Qingdao Agricultural University, 9-18.

Gijs, L., Chevance, F., Jerkovic, V., and Collin, S. (2002). How low ph can intensify $\beta$-damascenone and dimethyl trisulfide production through beer aging. J. Agric. Food Chem. 50, 5612-5616. doi: 10.1021/jf020563p

Gu, C., Yang, X., Al-Attala, M., Abid, M., Phyo, S., Zang, H., et al. (2020). First report of pomegranate fruit rot caused by Botryosphaeria dothidea in Anhui Province of China. Plant Dis. 104, 2736-2736. doi: 10.1094/PDIS-04-20-0790PDN

Guan, Y., Chang, R., Liu, G., Wang, Y., Wu, T., Han, Z., et al. (2015). Role of lenticels and microcracks on susceptibility of apple fruit to Botryosphaeria dothidea. Eur. J. Plant Pathol. 143, 317-330. doi: 10.1007/s10658-015-0682-Z

Hu, G., Sheng, C., Mao, R., Ma, Z., Lu, Y., and Wei, D. (2013). Essential oil composition of Allium tuberosum seed from China. Chem. Nat. Compounds 48 , 1091-1093. doi: 10.1007/s10600-013-0476-5

Huang, Y. H., Wang, R. C., Li, C. H., Zuo, C. W., Wei, Y. R., Zhang, L., et al. (2012). Control of Fusarium wilt in banana with Chinese leek. Eur. J. Plant Pathol. 134, 87-95. doi: 10.1007/s10658-012-0024-3

Huang, Y., Meng, L. L., Liu, J., and Wang, C. X. (2019b). First report of shoot canker on apricot caused by Botryosphaeria dothidea in Shandong Province of China. Plant Dis. 103, 2945-2946. doi: 10.1094/PDIS-03-19-0522-PDN

Huang, Y., Meng, L., Liu, J., and Wang, C. X. (2019a). First report of Botryosphaeria dothidea causing shoot canker on mulberry in China. Plant Dis. 103, 1788-1789. doi: 10.1094/PDIS-01-19-0183-PDN

Hughes, B., and Lawson, L. (1991). Antimicrobial effects of Allium sativum L. (Garlic), Allium ampeloprasum L. (Elephant Garlic), and Allium cepa L.(Onion), garlic compounds and commercial garlic supplement products. Phytother. Res. 5, 154-158. doi: 10.1002/ptr.2650050403

Ismail, S., Jalilian, F. A., Talebpour, A. H., Zargar, M., Shameli, K., Sekawi, Z., et al. (2013). Chemical composition and antibacterial and cytotoxic activities of Allium hirtifolium Boiss. BioMed Res. Int. 2013:696835. doi: 10.1155/2013/ 696835
Isogai, A., Kanda, R., Hiraga, Y., Nishimura, T., Iwata, H., and Goto-Yamamoto, N. (2009). Screening and identification of precursor compounds of dimethyl trisulfide (DMTS) in Japanese sake. J. Agric. Food Chem. 57, 189-195. doi: $10.1021 /$ jf802582p

Jiao, Z., Tian, Y., Cao, Y., Wang, J., Zhan, B., Zhao, Z., et al. (2021). A novel pathogenicity determinant hijacks maize catalase 1 to enhance viral multiplication and infection. New Phytol. 230, 1126-1141. doi: 10.1111/nph. 17206

Jin, Z., Li, L., Zheng, Y., and An, P. (2021). Diallyl disulfide, the antibacterial component of garlic essential oil, inhibits the toxicity of Bacillus cereus ATCC 14579 at sub-inhibitory concentrations. Food Control. 126:108090. doi: 10.1016/ j.foodcont.2021.108090

Karunanidhi, A., Ghaznavi-Rad, E., Jeevajothi Nathan, J., Abba, Y., van Belkum, A., and Neela, V. (2017). Allium stipitatum extract exhibits in vivo antibacterial activity against methicillin-resistant Saphylococcus aureus and accelerates burn wound healing in a full-thickness murine burn model. Evidence Based Complementary Alternat. Med. eCAM 2017:1914732. doi: 10.1155/2017/ 1914732

Kaur, R., Kalia, A., Lore, J. S., Kaur, A., Yadav, I., Sharma, P., et al. (2021). Trichoderma sp. endochitinase and $\beta$-1,3-glucanase impede Rhizoctonia solani growth independently, and their combined use does not enhance impediment. Plant Pathol. [Preprint]. doi: 10.1111/ppa.13381

Korukmez, N., Yildiz, F., Yayla, S., Gencer, R., and Akpinar, O. (2019). First report of fruit rot caused by Botryosphaeria dothidea on olive in Turkey. J. Plant Pathol. 102, 537-537. doi: 10.1007/s42161-019-00429-w

Kumar, R., Kumari, K., Hembram, K. C., Kandha, L., and Bindhani, B. K. (2018). Expression of an endo $\alpha-1,3$-Glucanase gene from Trichoderma harzianum in rice induces resistance against sheath blight. J. Plant Biochem. Biotechnol. 28, 84-90. doi: 10.1007/s13562-018-0465-7

Kurt, S., Gunes, U., and Soylu, E. M. (2011). In vitro and in vivo antifungal activity of synthetic pure isothiocyanates against Sclerotinia sclerotiorum. Pest Manage. Sci. 67, 869-875. doi: 10.1002/ps.2126

Li, J., Fu, S., Fan, G., Li, D., Yang, S., Peng, L., et al. (2021). Active compound identification by screening 33 essential oil monomers against Botryosphaeria dothidea from postharvest kiwifruit and its potential action mode. Pest. Biochem. Physiol. 2021:104957. doi: 10.1016/j.pestbp.2021.104957

Li, Q., Qin, X., Qi, J., Dou, W., Dunand, C., Chen, S., et al. (2020). CsPrx25, a class III peroxidase in Citrus sinensis, confers resistance to citrus bacterial canker through the maintenance of ROS homeostasis and cell wall lignification. Horticult. Res. 7:192. doi: 10.1038/s41438-020-00415-9

Liao, M., Ren, X., Gao, Q., Liu, N., Tang, F., Wang, G., et al. (2021). Antifungal activity of moso bamboo (Phyllostachys pubescens) leaf extract and its development into a botanical fungicide to control pepper phytophthora blight. Sci. Rep. 11:4146. doi: 10.1038/s41598-021-83598-y

Livak, K. J., and Schmittgen, T. D. (2001). Analysis of relative gene expression data using real-time quantitative PCR and the 2- ${ }^{\Delta C T}$ method. Methods 25, 402-408. doi: 10.1006/meth.2001.1262

Munday, R., Munday, J. S., and Munday, C. M. (2003). Comparative effects of mono-, di-, tri-, and tetrasulfides derived from plants of the Allium family: redox cycling in vitro and hemolytic activity and phase 2 enzyme induction in vivo. Free Radic. Biol. Med. 34, 1200-1211. doi: 10.1016/s0891-5849(03) 00144-8

Natasya-Ain, R., Eirna-Liza, N., Jasmin, M., and Karim, M. (2018). Antibacterial activity of garlic extracts on fish pathogenic bacteria. J. Environ. Biol. 39, 808-812. doi: 10.22438/jeb/39/5(SI)/25

Navarro-Gonzalez, S. S., Ramirez-Trujillo, J. A., Pena-Chora, G., Gaytan, P. Roldan-Salgado, A., Corzo, G., et al. (2019). Enhanced tolerance against a fungal pathogen and insect resistance in transgenic tobacco plants overexpressing an endochitinase gene from Serratia marcescens. Int. J. Mol. Sci. 20:ijms20143482. doi: 10.3390/ijms20143482

Ortega-Ramirez, L. A., Silva-Espinoza, B. A., Vargas-Arispuro, I., GonzalezAguilar, G. A., Cruz-Valenzuela, M. R., Nazzaro, F., et al. (2017). Combination of Cymbopogon citratus and Allium cepa essential oils increased antibacterial activity in leafy vegetables. J. Sci. Food Agricult. 97, 2166-2173. doi: 10.1002/ jsfa. 8025

Pan, J., Hao, X., Yao, H., Ge, K., Ma, L., and Ma, W. (2019). Matrine inhibits mycelia growth of Botryosphaeria dothidea by affecting membrane permeability. J. For. Res. 30, 1105-1113. doi: 10.1007/s11676-019-00883-3 
Pan, J., Yang, Y., Zhang, R., Yao, H., Ge, K., Zhang, M., et al. (2017). Enrichment of chelidonine from Chelidonium majus L. using macroporous resin and its antifungal activity. J. Chromatogr. 1070, 7-14. doi: 10.1016/j.jchromb.2017. 10.029

Qiu, F., Xu, G., Zhou, J., Zheng, F.-Q., Zheng, L., Miao, W., et al. (2020). First report of Botryosphaeria dothidea causing stem-end rot in avocado (Persea americana) in China. Plant Dis. 104, 286-287. doi: 10.1094/PDIS-07-19-1439-PDN

Rasooli, I., Rezaei, M. B., and Allameh, A. (2006). Growth inhibition and morphological alterations of Aspergillus niger by essential oils from Thymus eriocalyx and Thymus x-porlock. Food Control. 17, 359-364. doi: 10.1016/j. foodcont.2004.12.002

Rattanachaikunsopon, P., and Phumkhachorn, P. (2009). Potential of Chinese chive oil as a natural antimicrobial for controlling Flavobacterium columnare infection in Nile tilapia Oreochromis niloticus. Fisher. Sci. 75, 1431-1437. doi: 10.1007/s12562-009-0171-4

Sandhu, J. S., Nayyar, S., Kaur, A., Kaur, R., Kalia, A., Arora, A., et al. (2019). Foot rot tolerant transgenic rough lemon rootstock developed through expression of beta-1,3-glucanase from Trichoderma spp. Plant Biotechnol. J. 17, 2023-2025. doi: $10.1111 /$ pbi.13152

Santos, C., Nogueira, F. C. S., Domont, G. B., Fontes, W., Prado, G. S., Habibi, P., et al. (2019). Proteomic analysis and functional validation of a Brassica oleracea endochitinase involved in resistance to Xanthomonas campestris. Front. Plant Sci. 10:414. doi: 10.3389/fpls.2019.00414

Shahriari, M., Hemmati, S., Zangeneh, A., and Zangeneh, M. M. (2019). Biosynthesis of gold nanoparticles using Allium noeanum Reut. ex Regel leaves aqueous extract; characterization and analysis of their cytotoxicity, antioxidant, and antibacterial properties. Appl. Organometal. Chem. 33:5189. doi: 10.1002/ aoc. 5189

Singh, H. R., Hazarika, P., Agarwala, N., Bhattacharyya, N., Bhagawati, P., Gohain, B., et al. (2018). Transgenic tea over-expressing Solanum tuberosum endo-1,3beta-d-glucanase gene conferred resistance against blister blight disease. Plant Mol. Biol. Rep. 36, 107-122. doi: 10.1007/s11105-017-1063-x

Song, Y., Li, L., Li, C., Lu, Z., Men, X., and Chen, F. (2018). Evaluating the sensitivity and efficacy of fungicides with different modes of action against Botryosphaeria dothidea. Plant Dis. 102, 1785-1793. doi: 10.1094/pdis-01-18-0118-re

Sorlozano-Puerto, A., Albertuz-Crespo, M., Lopez-Machado, I., Gil-Martinez, L., Ariza-Romero, J. J., Maroto-Tello, A., et al. (2020). Antibacterial and antifungal activity of propyl-propane-thiosulfinate and propyl-propane-thiosulfonate, two organosulfur compounds from Allium cepa: in vitro antimicrobial effect via the gas phase. Pharmaceuticals 14:h14010021. doi: 10.3390/ph14010021

Soylu, E. M., Kurt, S., and Soylu, S. (2010). In vitro and in vivo antifungal activities of the essential oils of various plants against tomato grey mould disease agent Botrytis cinerea. Int. J. Food Microbiol. 143, 183-189. doi: 10.1016/j.ijfoodmicro. 2010.08.015

Soylu, E. M., Soylu, S., and Kurt, S. (2006). Antimicrobial activities of the essential oils of various plants against tomato late blight disease agent Phytophthora infestans. Mycopathologia 161, 119-128. doi: 10.1007/s11046-005-0206-Z

Sun, X., Pan, B., Wang, Y., Xu, W., and Zhang, S. (2020). Exogenous calcium improved resistance to Botryosphaeria dothidea by increasing autophagy activity and salicylic acid level in pear. Mol. Plant Microbe Interact. 33, 11501160. doi: 10.1094/MPMI-04-20-0101-R

Tang, W., Ding, Z., Zhou, Z. Q., Wang, Y. Z., and Guo, L. Y. (2012). Phylogenetic and pathogenic analyses show that the causal agent of apple ring rot in China is Botryosphaeria dothidea. Plant Dis. 96, 486-496. doi: 10.1094/PDIS-08-11-0635

Wang, L., Hou, H., Zhou, Z., Tu, H., and Yuan, H. (2021). Identification and detection of Botryosphaeria dothidea from kiwifruit (Actinidia chinensis) in China. Plants 10:lants10020401. doi: 10.3390/plants10020401

Wang, X., Zhang, X., Li, M., Ji, X., Feng, C., and Wang, F. (2020). First report of ficus carica bot rot caused by Botryosphaeria dothidea in China. Plant Dis. 104, 1869-1879. doi: 10.1094/PDIS-09-19-2039-PDN

Xiao, L., Zhou, Y. M., Zhang, X. F., and Du, F. Y. (2018). Notopterygium incisum extract and associated secondary metabolites inhibit apple fruit fungal pathogens. Pest. Biochem. Physiol. 150, 59-65. doi: 10.1016/j.pestbp.2018.07.001
Yang, L., Zhu, Z., Zhang, J., Gao, Y., Wang, X., Liu, G., et al. (2020). Response of kiwifruit yield and fruit quality to chloride-containing fertilizers. Agronomy J. 112, 1012-1020. doi: 10.1002/agj2.20074

Yang, T., Qiu, L., Huang, W., Xu, Q., Zou, J., Peng, Q., et al. (2020). Chilli veinal mottle virus HCPro interacts with catalase to facilitate virus infection in Nicotiana tabacum. J. Exp. Bot. 71, 5656-5668. doi: 10.1093/jxb/ eraa304

Yang, Y., Jiang, R., Wang, H., Tian, Z., and Xie, C. (2020). StPOPA, encoding an anionic peroxidase, enhances potato resistance against Phytophthora infestans. Mol. Breed. 40:1. doi: 10.1007/s11032-019-1093-1

Yi, H., Chen, Y., Liu, J., Zhang, J., Guo, W., Xiao, W., et al. (2016). Extraction and separation of active ingredients in Schisandra chinensis (Turcz.) baill and the study of their antifungal effects. PLoS One 11:e0154731. doi: 10.1371/journal. pone. 0154731

Zangeneh, A., Zangeneh, M. M., and Moradi, R. (2019). Ethnomedicinal plantextract-assisted green synthesis of iron nanoparticles using Allium saralicum extract, and their antioxidant, cytotoxicity, antibacterial, antifungal and cutaneous wound-healing activities. Appl. Organometal. Chem. 34:5247. doi: 10.1002/aoc.5247

Zangeneh, M. M., Bovandi, S., Gharehyakheh, S., Zangeneh, A., and Irani, P. (2019). Green synthesis and chemical characterization of silver nanoparticles obtained using Allium saralicum aqueous extract and survey of in vitro antioxidant, cytotoxic, antibacterial and antifungal properties. Appl. Organometal. Chem. 33:4961. doi: 10.1002/aoc.4961

Zhang, L., Zhang, Q., Yang, P., Niu, Y., and Niu, W. (2019). First report of gummosis disease of sweet cherry caused by Botryosphaeria dothidea in China. Plant Dis. 103:1418-PDN

Zhang, Z., Xie, Y., Hu, X., Shi, H., Wei, M., and Lin, Z. (2018). Antifungal activity of monoterpenes against Botryosphaeria dothidea. Nat. Prod. Commun. 13:1934578X1801301. doi: 10.1177/1934578x1801301234

Zhao, G., Zhang, W., Zuo, C., and Huang, Y. (2017). Control effect of chinese leek extract and its main bioactive components on apple ring rot incidence. Chin. J. Biol. Control 33, 273-280. doi: 10.16409/j.cnki.2095-039x.2017. 02.019

Zhao, L., Phuong, L. T., Luan, M. T., Fitrianti, A. N., Matsui, H., Nakagami, H., et al. (2019). A class III peroxidase PRX34 is a component of disease resistance in Arabidopsis. J. General Plant Pathol. 85, 405-412. doi: 10.1007/s10327-01900863-9

Zhao, X., Zhang, G.-L., Li, B.-H., Xu, X.-M., Dong, X.-L., Wang, C.-X., et al. (2016). Seasonal dynamics of Botryosphaeria dothidea infections and symptom development on apple fruits and shoots in China. Eur. J. Plant Pathol. 146, 507-518. doi: 10.1007/s10658-016-0935-5

Zheng, Y., Wang, X., Liu, S., Zhang, K., Cai, Z., Chen, X., et al. (2018). The endochitinase of clonostachysrosea expression in Bacillus amyloliquefaciens enhances the Botrytis cinerea resistance of tomato. Int. J. Mol. Sci. 19:ijms19082221. doi: 10.3390/ijms19082221

Conflict of Interest: The authors declare that the research was conducted in the absence of any commercial or financial relationships that could be construed as a potential conflict of interest.

Publisher's Note: All claims expressed in this article are solely those of the authors and do not necessarily represent those of their affiliated organizations, or those of the publisher, the editors and the reviewers. Any product that may be evaluated in this article, or claim that may be made by its manufacturer, is not guaranteed or endorsed by the publisher.

Copyright (C) 2022 Sun, Duan, Liu, Fu and Huang. This is an open-access article distributed under the terms of the Creative Commons Attribution License (CC BY). The use, distribution or reproduction in other forums is permitted, provided the original author(s) and the copyright owner(s) are credited and that the original publication in this journal is cited, in accordance with accepted academic practice. No use, distribution or reproduction is permitted which does not comply with these terms. 